Provided for non-commercial research and education use. Not for reproduction, distribution or commercial use.

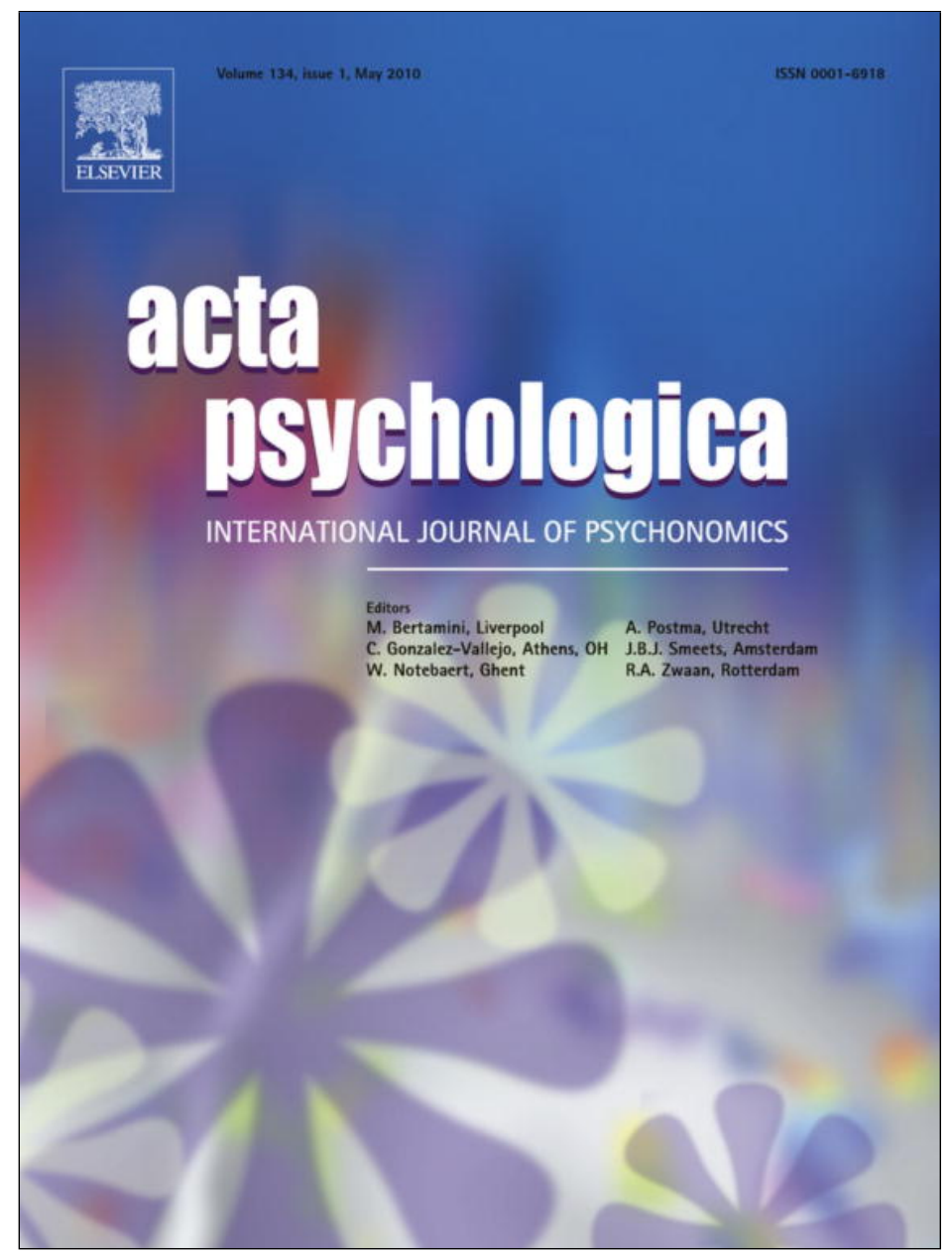

This article appeared in a journal published by Elsevier. The attached copy is furnished to the author for internal non-commercial research and education use, including for instruction at the authors institution and sharing with colleagues.

Other uses, including reproduction and distribution, or selling or licensing copies, or posting to personal, institutional or third party websites are prohibited.

In most cases authors are permitted to post their version of the article (e.g. in Word or Tex form) to their personal website or institutional repository. Authors requiring further information regarding Elsevier's archiving and manuscript policies are encouraged to visit:

http://www.elsevier.com/copyright 


\title{
Naïve predictions of motion and orientation in mirrors: From what we see to what we expect reflections to do
}

\author{
Ugo Savardi $^{a}$, Ivana Bianchi ${ }^{\mathrm{b}, *}$, Marco Bertamini $^{\mathrm{c}}$ \\ ${ }^{a}$ Department of Psychology and Cultural Anthropology, University of Verona, Italy \\ ${ }^{\mathrm{b}}$ Department of Educational Sciences, University of Macerata, P.le Bertelli 1, 62100 Macerata, Italy

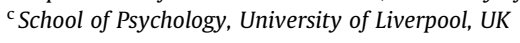

\section{A R T I C L E I N F O}

\section{Article history:}

Received 10 April 2009

Received in revised form 15 November 2009

Accepted 16 November 2009

Available online 16 December 2009

\section{PsycINFO classification:}

2340

2323

Keywords:

Mirror image

Naïve optics

Naïve physics

\begin{abstract}
A B S T R A C $T$
The study aimed to investigate naïve beliefs regarding the dynamic and static behavior of reflections. In the first three experiments, participants in the study made predictions about the correspondence between real and reflected movements or about the orientation of the reflection of a static object placed in front of a mirror. In Experiments 1 and 2, paper-and-pencil tasks were used and in Experiment 3 participants were asked to make their predictions while imagining that they were facing a mirror. Results revealed that a percentage of undergraduates (ranging from $25 \%$ to $35 \%$ ) were unable to make correct predictions. We classified the errors into types and found that responses either conform to the belief that reflections do the same or that they do the opposite. This suggests an oversimplification of the geometry of mirror reflections in two directions: participants either generalize what they see when movements are parallel to the mirror or what they see when movements are orthogonal to the mirror. Findings from Experiment 4 confirmed that these two expectations fit in with what people perceive in mirrors. Findings from Experiment 5 confirmed that this is also in agreement with the relationship perceived when looking at similar movements and orientations "outside" mirrors.
\end{abstract}

(c) 2009 Elsevier B.V. All rights reserved.

\section{Beliefs regarding motion in naïve physics: errors, explanations, and foundations}

There is a body of research on the commonsense beliefs that people hold about the types of motion studied in classical mechanics: the trajectories and speed of falling objects (Bozzi, 1959; Kaiser, Proffitt, \& McCloskey, 1985; McCloskey, Washburn, \& Felch, 1983; Runeson, 1974; Shanon, 1976), projectiles (Hecht \& Bertamini, 2000; McCloskey, 1983; McCloskey, Caramazza, \& Green, 1980; Shaffer \& McBeath, 2005), pendula (Bozzi, 1958; Frick, Huber, Reips, \& Krist, 2005; Pittenger, 1989) and wheel dynamics (Proffitt \& Gilden, 1989; Profitt, Kaiser, \& Whelan, 1990). These beliefs form the basis of what is known as naïve physics or intuitive physics (McCloskey, 1983; see Proffitt, 1999 and Proffitt \& Kaiser, 2003 for brief overviews). The research revealed systematic errors made by adults when predicting simple motions. There have been debates on the origin of these errors and of naïve beliefs concerning motion in general. Are they founded on what we perceive (e.g., Bozzi, 1958, 1959; McCloskey et al., 1983; Pittenger, 1989)? Do

\footnotetext{
* Corresponding author: Tel.: +39 07332584320 (office), +39 3383808943 (mobile).

E-mail addresses: ugo.savardi@univr.it (U. Savardi), ivana.bianchi@unimc.it (I. Bianchi), m.bertamini@liverpool.ac.uk (M. Bertamini).
}

people imagine prototypes of actions which they mentally run through (e.g., Yates et al., 1988)? Or do these beliefs mean that they manifest implicit theories or mental models that people have in mind (e.g., McCloskey, 1983; Proffitt \& Gilden, 1989; Profitt et al., 1990)?

The classic explanation is that people develop implicit theories of motion which do not conform to the principles of Newtonian physics. This is what was suggested in the initial milestone studies by McCloskey and colleagues and it became, with few exceptions, the leading mindset.

A different perspective, which - in our view - has been underestimated, was suggested by Yates et al. (1988). They interviewed adults who had been asked to solve these types of motion problems and they found that the solutions were the result of mental reenactment of prototypes, rather than deductions from a theory. This was reported by the participants themselves, but was also confirmed by the fact that when problems were described in a different way, this produced a variation in the reenactment and thus a change in the response. For instance, a variation of the classic C-tube problem asked people to directly focus on the effect of spinning on a ball ("imagine being inside the ball, and imagine the forces you would feel as the ball is forced by the outer wall of the tube to travel a curved trajectory..."); the number of participants predicting a curved path was higher in this condition than in the classic condition. 
This suggested that people do not derive their responses from a set of explanatory principles or laws describing an abstract and general model of the phenomenon (i.e., a theory), but use more local and concrete models, derived by imagining specific situations. "The model of the world thereby allows complex imaginary events to be experienced as if they actually had occurred, an extension of Shepard's finding that subjects can experience the rotation of mental objects (see Shepard \& Cooper, 1982, for a review). Just as subjects can answer questions concerning form by enacting the rotation of a random form, they might answer a question about motion, such as the motion of a sling, by drawing on past experience (in the form of prototype events and objects) to create an enactment of the event implied by the question..." (Yates et al., 1988, p. 253). When asked to predict an event, people would recall - out of the available set of event prototypes they have in mind - that prototype which best accounts for the problem or that which can be most readily modified to account for it. The salient features of a dynamic event can affect the way people think about a class of similar events. This hypothesis is central to the studies presented in this paper.

\section{Beliefs regarding motion in naïve optics: errors, explanations, and foundations}

Comparatively few studies have investigated people's beliefs about reflections and only some of them have focused on motion, in most cases indirectly (Bertamini, Lawson, \& Liu, 2008; Bertamini \& Parks, 2005; Bertamini, Spooner, \& Hecht, 2003; Bianchi \& Savardi, 2008; Bianchi, Savardi, \& Bertamini, 2008; Croucher, Bertamini, \& Hecht, 2002; Hecht, Bertamini, \& Gamer, 2005; Lawson \& Bertamini, 2006; Savardi \& Bianchi, 2005). One of the errors that emerged in predictive tasks and which indirectly has to do with imagining the dynamic behavior of mirrors, concerned predictions about where on a mirror surface observers would see themselves or a target object.

Using a paper-and-pencil task, Bertamini et al. (2003) found that, when participants were asked to imagine a person entering a room and moving parallel to a mirror surface and to predict where the reflection would appear, they frequently expected reflections to appear on the opposite side with respect to the direction in which the person was actually moving (Fig. 1). In one condition a drawing showed a mirror on the wall and a person walking parallel to the mirror; another condition showed the person moving vertically in a lift in front of the mirror; in a further condition, the mirrors were positioned either on the floor or on the ceiling, and the person was walking towards them. In the first condition, almost 50\% of the participants predicted (incorrectly) that the reflection would appear on the far side of the mirror. This error was found in $32 \%$ of cases in the lift condition. Performance improved also in the case of the mirror lying on the floor or attached to the ceiling (the error was found in around $20 \%$ and $30 \%$ of predictions, respectively).

There are some errors that people make about mirrors that are almost universal. For instance, people are unable to judge the size of a projected reflection on the surface of a mirror (Lawson, Bertamini, \& Liu, 2007). The error in Fig. 1 was only present in a minority of cases, but nevertheless, we believe it is interesting because it is qualitative rather than quantitative: the positioning of the image is completely reversed.

How might this error be explained?

\subsection{Competing explanations for the location error}

\subsubsection{Familiarity}

Since people always see their reflection appearing on the near side of a mirror when they move parallel to it, familiarity cannot
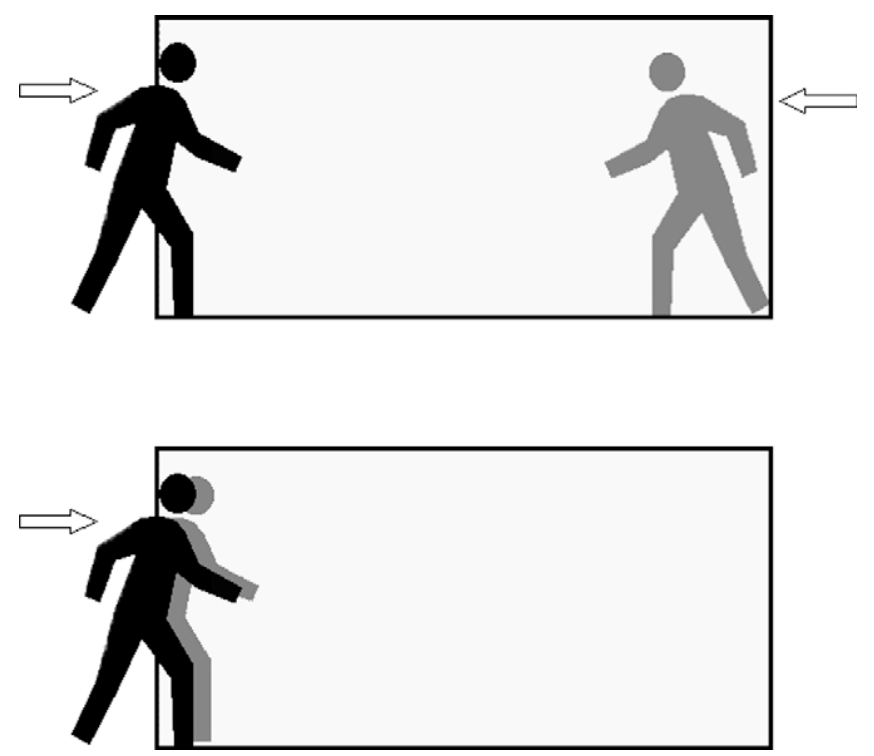

Fig. 1. Schematic illustration of the error found by Bertamini et al. (2003). If asked to imagine a person (here represented by the black silhouette) approaching a mirror, almost $50 \%$ of undergraduate students predict that the reflection (here, the gray silhouette) would appear on the opposite side (top) rather than on the near side (bottom).

be the underlying cause of the location error (Fig. 1). Moreover, people have more experience approaching mirrors on walls rather than on the floor, but in the latter case, errors are less frequent.

\subsubsection{Misunderstanding the task}

The question asked was simple and there are no reasons to consider it misleading, so it is unlikely that errors originate from a misunderstanding.

\subsubsection{The implicit theory of rotation}

Bertamini et al. (2003) suggested that the cause might be the false belief that the transition from actual to reflected world consists of a $180^{\circ}$ rotation around a vertical axis. This suggests that some kind of general law is being applied regarding reflections. However, in the floor condition (where there were few errors) people did not seem to expect a $180^{\circ}$ rotation. Moreover, people do recognize a problem with images where the reflection has been reversed left-right around its vertical axis, i.e., a transformation consistent with a rotation of the real scene (Bertamini et al., 2003, Experiment 3). Neither of these results can be explained by the rotation hypothesis.

\subsubsection{Identity-opposition}

An alternative interpretation has been suggested by Bianchi et al. (2008), based on their study of the relationships perceived by adults looking at their own or other people's reflections. They suggested that the location error might be related to their findings that the exocentric spatial opposition of the reflected body with respect to the real body is a salient characteristic of reflections in plane mirrors. Participants facing a mirror rarely noticed the egocentric opposition - e.g., their (real) right hand is the left-hand in the mirror. This type of frontal reflection was strongly associated with a sense of identity. However, participants frequently (in $80 \%$ of cases) reported that the reflection had an opposite posture, using the exocentric frame of reference to recognize this ("I'm facing north, while the reflection is facing south"). With reflections formed on a single plane mirror set horizontally on the floor, participants more frequently reported a general perception of opposi- 
tion due to the reversed exocentric up-down orientation, while they never mentioned the egocentric left-right reversal. Furthermore, with familiar gestures - e.g., arm stretched towards the mirror - in more than $70 \%$ of cases participants described the reflection as performing a non-identical gesture and described it as opposite. An analysis in terms of egocentric versus exocentric frames of reference confirmed that, independently of whether reflections were on mirrors set in front, to the side, or under their feet, gestures pointing in an opposite exocentric direction with respect to the real body were recognized as opposite gestures.

On the basis of these results, Bianchi et al. (2008, p. 685) suggested the following: "The mistaken expectation that the reflection would appear on the opposite side of the mirror could be explained as an erroneous generalization of the exocentric structure of opposition that usually characterizes our perception of reflections. When we look at ourselves or at any other person or object approaching a vertical mirror from various angles, we see that the reflection is moving simultaneously in an identical exocentric direction and in the opposite direction (see Fig. 2). These two components characterize any movement towards mirrors on a wall, with only two exceptions. The first is when approaching it by walking orthogonally towards it (Fig. 2d). In this case only the opposition component is present. The second is when approaching it walking parallel to the mirror (Fig. 2c) - as in the condition studied by Bertamini et al. (2003) - where the opposition component disappears in favor of the identity-of-direction component alone."

In this paper we start from this last consideration, i.e., that the perception of what happens in a mirror is constrained by these two components (identity and opposition). We will test the hypothesis that these principles constrain the way in which people think of mirror phenomena when not directly observing a mirror.

\section{General aims of the research}

Our general aim was to understand people's knowledge of the correspondence between the movement (or orientation) of an object and the movement (or orientation) of its reflection. In particular, we aimed to determine whether:

(1) Errors are consistent across different tasks or, conversely, change with the superficial structure of the task. This last possibility is a problem for researchers who think of people's naïve beliefs in terms of a single internal model. It is, however, compatible with and even necessary for the hypothesis that people, for certain types of motion, have in mind various dynamic prototypes and use their memory and imagination to retrieve these.

(2) The structure of the errors manifests a geometric model of reflections which is based on identity or oppositional pat- terns rather than on rotational models. This hypothesis is based on the idea cited by Bianchi et al. (2008) above.

If we express the idea in terms of vectors and Cartesian axes $x, y$, and $z$ (and focusing, initially, on movements), when the vector corresponding to the real motion is parallel to a mirror (for both $x$ and $y$ ) the reflected vector points in the identical direction (Fig. 2c). When the vector corresponding to the real motion is orthogonal to the mirror (along $z$ ), the reflected vector points in the opposite direction (Fig. 2d). In all other cases, that is when the movement towards (or away from) the mirror is oblique, the reflected movement is the resultant (in vectorial terms) of the $x, y$, and $z$ components (Fig. 2a and b), and in a sense both identity and opposition are present. Movements which are parallel and orthogonal to the mirror are two special cases, where one of the vectors is null and where the geometry of the correspondence between real and reflected worlds is particularly simple and straightforward. Describing the spatial characteristics of reflections in terms of vectors which are identically oriented or oriented in the opposite direction complies not only with what we consider to be the essential spatial structure of the phenomena, but also with the relationship perceived by participants when comparing their own orientation or gestures with the reflected orientation or gestures (as has been at least in part demonstrated by Bianchi et al., 2008).

If, when asked to predict the dynamics of motion reflected in mirrors, people do not refer to the geometry as described above in terms of three axes $(x, y$, and $z$ ), but instead recall only the two easiest patterns (orthogonal movement and parallel movement to the mirror) or overall impressions of identity and opposition, two errors can be expected:

(1) a generalization of the identity pattern - maximally evident when looking at someone/something moving parallel to the mirror surface; this might lead participants to predict that, in general, reflections do the same;

(2) a generalization of the opposition pattern - which is maximally evident when looking at someone/something moving orthogonal to the mirror surface; this might lead participants to predict that, in general, reflections do the opposite.

Experiments 1 to 3 were designed to test whether, in tasks which involve predicting the correct correspondence between movements (or orientations of an object) and their reflections, errors comply with these two rules rather than with the hypothesis that people think of this correspondence in terms of rotation. Experiments 4 and 5 aimed to find further proof that these two rules are in line with the salient relationships which are perceived by people when looking at an object (or movement) and at its reflection or at two objects (or movements) which are spatially

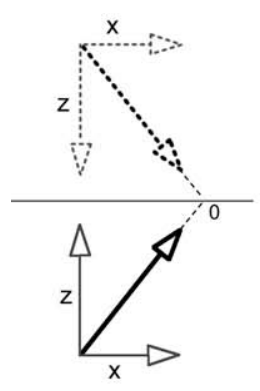

(a)

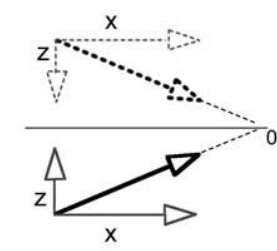

(b)

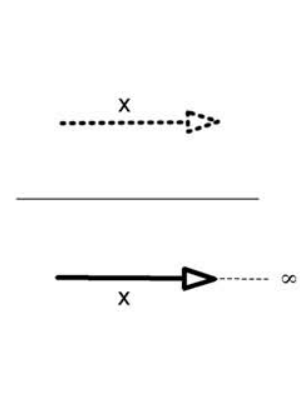

(c)

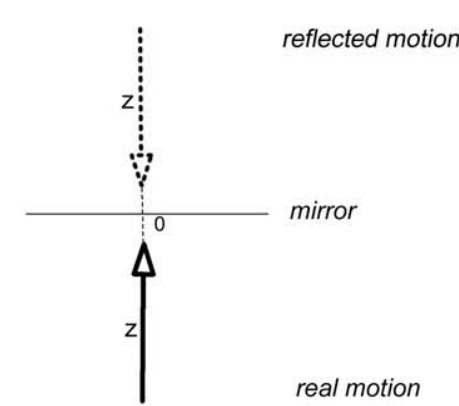

(d)

Fig. 2. Representation (aerial view) of the relationship between real movements (hard lines) and virtual movements (broken lines) in a two-dimensional Cartesian plane ( $x$ and $z$ ), described in terms of component and resultant vectors. The $y$ axis has not been considered for simplicity. When the motion is parallel to the mirror along the $x$ axis (c), but the same holds along $y$, the orthogonal component $(z)$ is null. Conversely, when the motion is orthogonal towards the mirror (d), the parallel component $(x)$ is null. 
oriented as if they were an object and its corresponding virtual copy.

\section{Experiment 1: predicting the motion of reflected objects from the motion of real objects}

In the first experiment, we asked people to predict the direction of the reflection of a moving object. To keep the task simple, we focused only on movements along a straight line. We considered different angles of incidence (from parallel to the mirror, i.e., $0^{\circ}$, to orthogonal to the mirror, i.e., $90^{\circ}$, through $22.5^{\circ}, 45^{\circ}$ and $67.5^{\circ}$ ) and two directions of motion (movements towards or away from the mirror). Participants were asked to predict the entire trajectory of the movement.

The aim of the study was to test whether there are more correct responses when movements are parallel or orthogonal to a mirror, that is when the geometry conforms, respectively, to the simple identity rule (reflections do the same) or the simple opposition rule (reflections do the opposite), and whether the errors show a generalization of the identity or opposition rules.

\subsection{Method}

\subsubsection{Participants}

Eighty-three undergraduate students doing an introductory course in General Psychology at the University of Verona (63 females).

\subsubsection{Procedure}

Each participant was presented with nine sheets of paper, in a random order, each showing a schematic drawing of a mirror with an arrow representing a movement (see Fig. 3). They were asked: (a) to draw, on the other side of the mirror, an arrow corresponding to the predicted motion of the reflection; (b) to rate how confident they were about the response, using an 11-point scale (0-10) printed at the bottom of each sheet.

\subsubsection{Materials}

Nine sheets of paper, each showing a schematic drawing representing an aerial view of a mirror and an arrow representing a movement. Stimuli varied for Angle of incidence $\left(0^{\circ}, 22.5^{\circ}, 45^{\circ}\right.$, $67.5^{\circ}$, and $90^{\circ}$ ) and Direction of motion (towards the mirror, or convergent, and away from the mirror, or divergent). For the condition of motion parallel to the mirror (angle of incidence $=0^{\circ}$ ), we arbitrarily chose one of the two possible directions (in this case, right).

\subsection{Results}

The initial analysis revealed that responses maintained the same Angle of incidence as the real motion (we interpreted deviations up to $\pm 5^{\circ}$ in the angles as due to imprecision in drawing). Errors, when present, concerned two aspects; we therefore classified participants' responses according to two criteria.

The first criterion was the Type of transformation, defined on the basis of the two components as described in Fig. 2, one parallel and the other orthogonal to the mirror. In any case, the orthogonal component is always opposite in the reflection with respect to the real movement, whereas the parallel component is always identical to the real movement. In two of the possible conditions, one of the two components is null, namely for movements parallel to the mirror $\left(0^{\circ}\right)$ where the orthogonal component is null, and for movements orthogonal to the mirror $\left(90^{\circ}\right)$ where the parallel component is null.

With these rules in mind, responses were classified as shown in Fig. 4. Namely:

(i) Correct IO: parallel component: Identical, orthogonal component: Opposite;

(ii) Error type OO, parallel component: Opposite, orthogonal component: Opposite;

(iii) Error type II, parallel component: Identical, orthogonal component: Identical;

(iv) Error type OI, parallel component: Opposite, orthogonal component: Identical.

Both Error type $\mathrm{OO}$ and Error type II represent oversimplifications of the actual geometry of mirror reflections: one of the two components has not been taken into account and the expected behavior is thus thought of as the resultant of only one rather than two vectors. When that vector is the orthogonal component, responses manifest the belief that the reflection does the opposite (Error type 00); when it is the parallel component, responses manifest the belief that the reflection does the same (Error type II).

Error type OI, in contrast, does not manifest simplification but instead confusion between the two vectors: people seem to be aware that reflections can move in identical and, at the same time, opposite directions, but they invert the two components with respect to how they should be, i.e., they apply opposition to the parallel component and identity to the orthogonal component.

The second criterion used to classify responses regards Localization. Independently of the type of transformation, reflections were also classified as correct or incorrect in terms of localization (see

\footnotetext{
The drawing represents an aerial view of a mirror (the longer line) and the movement of an object in front of the mirror (the arrow). Draw, on the other side of the mirror, the movement of the reflected object as you would see it if you were standing where the gray circle is.
}

Rate, on the scale below, how confident you are about the response that you have just given (with 0: no confidence, 10 fully confident).

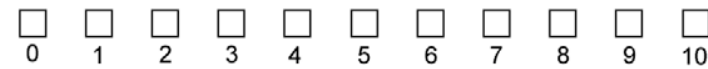

Fig. 3. Example of stimulus used for the paper-and-pencil task of Experiment 1 . In this example the movement is parallel to the mirror (or at $0^{\circ}$ angle of incidence). 


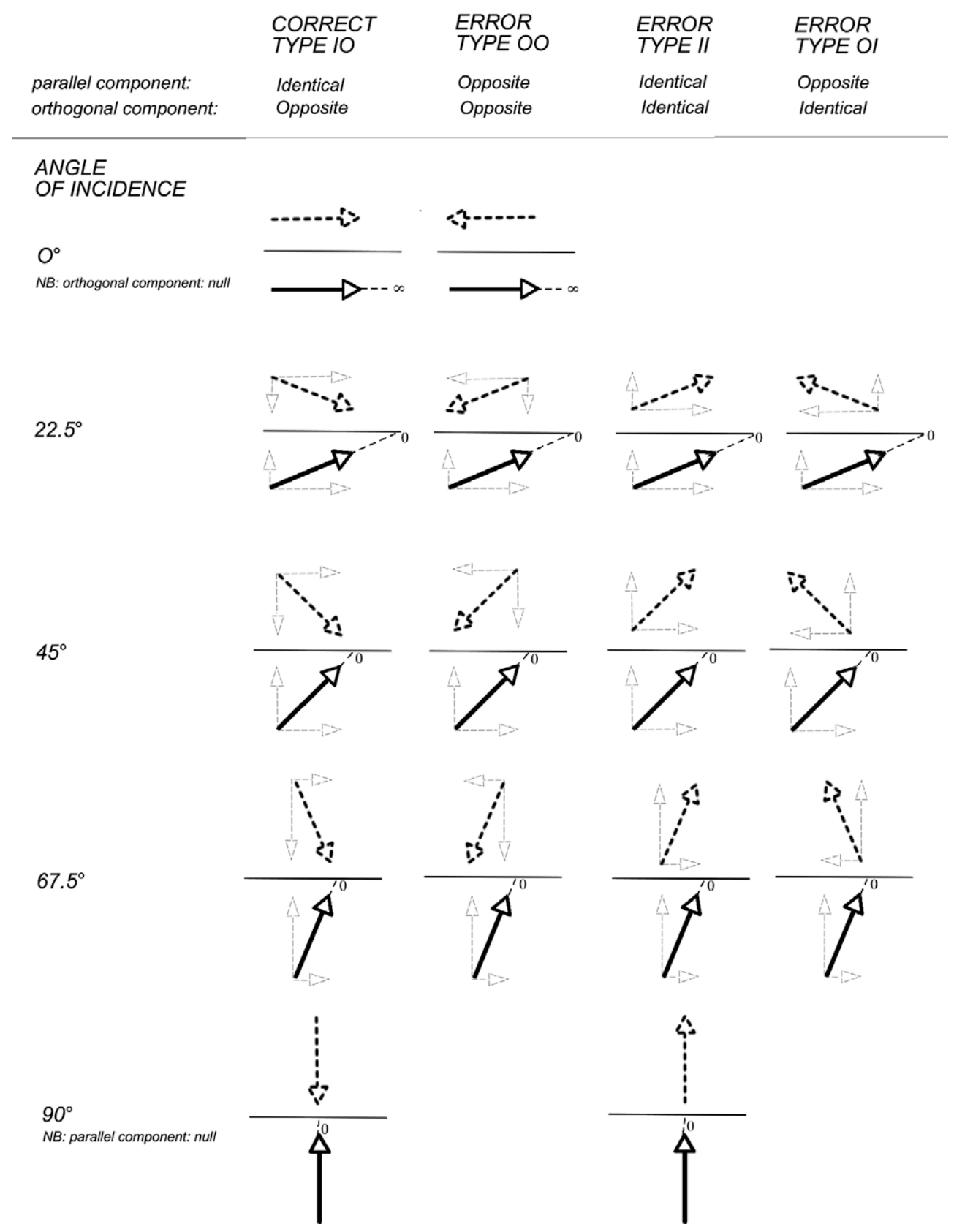

Fig. 4. Classification of participants' responses (in thick broken lines) in terms of type of transformation, based on the two components of motion, parallel and orthogonal to the mirror surface (represented in thin broken lines), for each of the angles of incidence.

Fig. 5). Note that the diagram for the correct response in Fig. 5 is wrong in terms of the direction of the arrow (i.e., in terms of type of transformation), but is localized in the correct area in the mirror. Two errors of localization were found: alignment of the reflected movement with one of the endpoints of the real movement (see endAlign error, in Fig. 5) and alignment of the reflected movement along the same line as the real movement (we will refer to this condition in terms of "coincidence of paths", and abbreviate it with coPaths, see Fig. 5).

We used generalized mixed-effect models and mosaic plots (Friendly, 1994; Friendly, 2000; Meyer, Zeileis, \& Hornik, 2006) to analyze the categorical data from Experiment 1 and did all the analyses using lme4, car and vcd packages of $\mathrm{R}$ version 2.9.1. Because the variable direction (away or towards the mirror) was not significant and was not present in any significant interaction, we will not discuss it in the results.
4.2.1. Total frequency of correct versus incorrect responses, in terms of type of transformation or localization (see Fig. 6, bar plots)

Almost $35 \%$ of the total number of responses $(n=256)$ were incorrect predictions about how the reflection would move. Errors in the type of transformation consisted in $16.9 \%$ of cases of Error type 00 , in $14.3 \%$ of cases of Error type II and in only $3.1 \%$ of cases of Error type OI. The localization of the reflection was correctly predicted in $72 \%$ of responses. Of the remaining $28 \%$ of incorrect localizations, 142 consisted of coPath errors (almost 20\%) and 62 (8.5\%) of endAlign errors.

\subsubsection{Interactions between variables}

Using generalized (linear) mixed-effect models, we tested various patterns of association between the variables. Only two-way interactions emerged from the analysis. We decided to use mosaic plots to study and present these interactions (see Fig. 6). A mosaic 

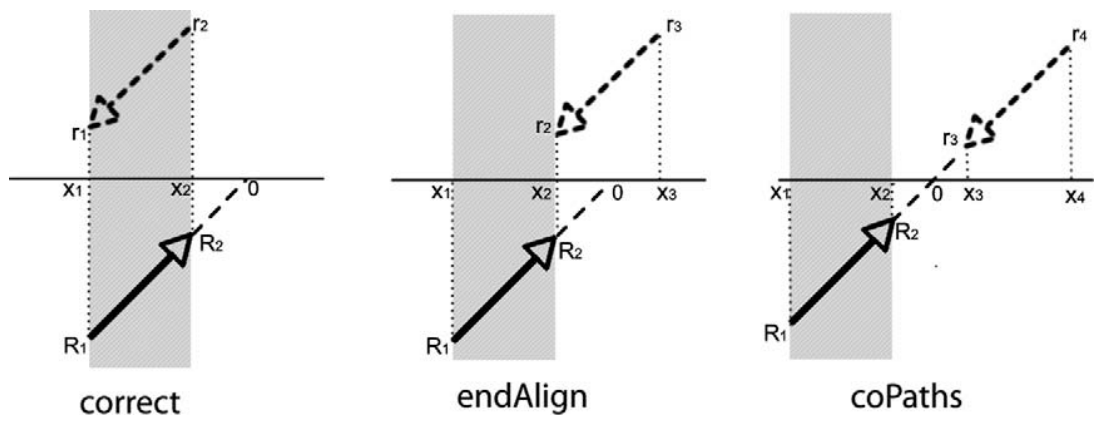

Fig. 5. Three different localizations of the reflected movement with respect to the real movement, applied to the same transformation (an example of Error type O0). Responses not falling in the gray region consist in incorrect localizations. The 3 types of localizations found can be described as follows: (1) correct (i.e., coincidence, in $x$, of both the endpoints of the real and reflected movements: $R_{1} \equiv r_{1}, R_{2} \equiv r_{2} ; 2$ ) endpoint alignment (i.e., coincidence, in $x$, of one of the endpoints: $R_{2} \equiv r_{2}$; 3 ) coincident paths (i.e., alignment of the endpoints $R_{1}, R_{2}, r_{3}, r_{4}$ along the line connecting the two movements - and which includes also the point where the movements "cross" the mirror surface).
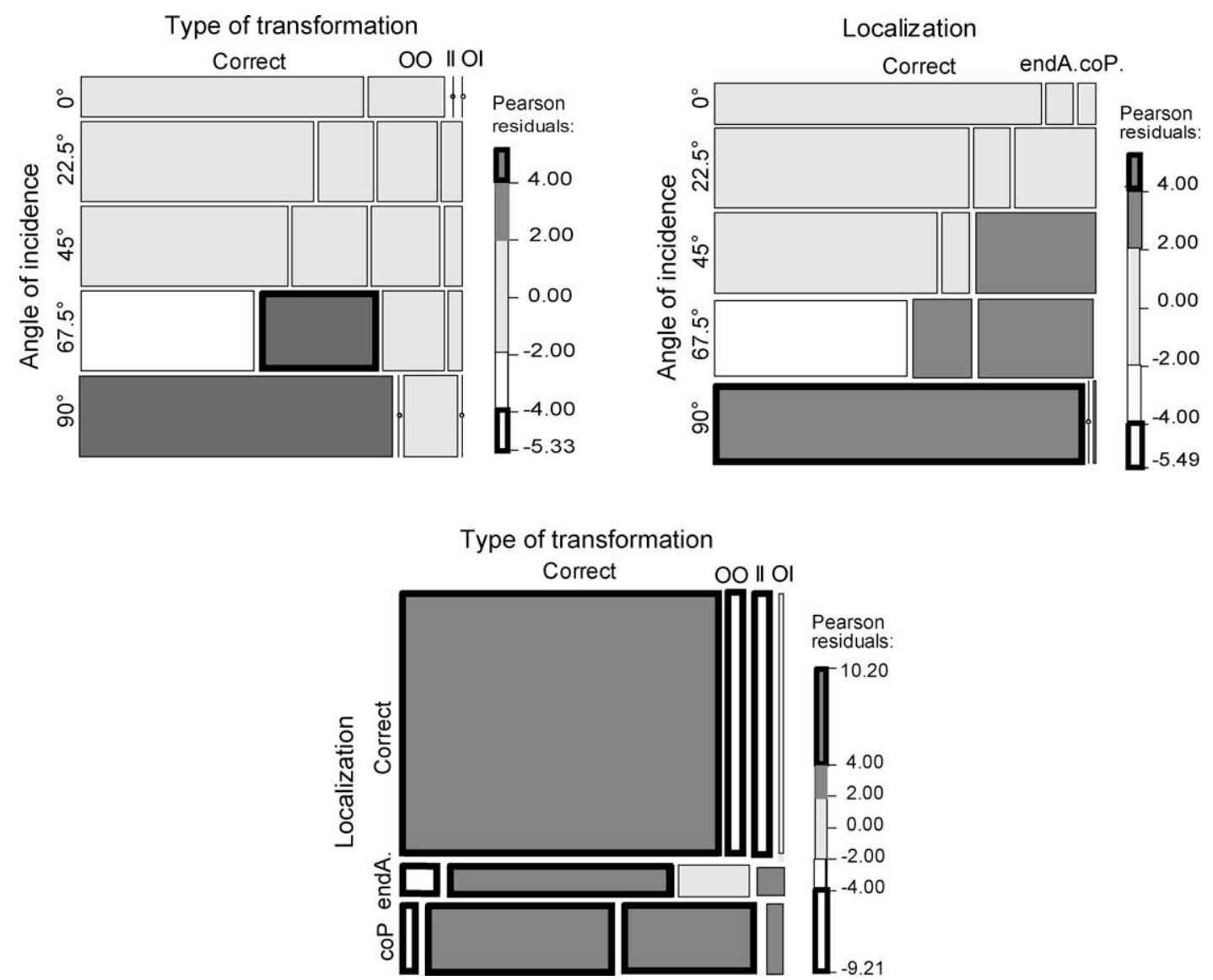

Fig. 6. Mosaics plots showing the two-way interactions between type of transformation and angle of incidence (top left), localization and angle of incidence (top right), type of transformation and localization (bottom). The interaction between the variables was significant in each of the three cases ( $p<0.0001$ ). The labels to the left and top of each mosaic refer to the variables (and their levels) and can be considered as the rows and columns of a contingency table; each tile corresponds to the crossing between one level of the left-hand side variable and one level of the top variable. In the bar to the right of each mosaic, dark gray and white tiles (with normal borders) correspond to Pearson residuals greater than $|2|$ (corresponding to $\alpha=0.05$ ); dark gray and white tiles (with thick borders) correspond to Pearson residuals greater than $|4|(\alpha=0.0001$ ).

plot represents (a) the observed frequencies of a contingency table by means of the size of the tiles, (b) the interaction between variables by means of the asymmetrical non-alignment of the tiles, and (c) the significance of the difference between observed and expected frequencies according to a specific model (in our case a log-linear model of independence between variables) by means of the color of the tiles. The color of the tiles corresponds to Pearson residuals, and the bars to the right of each mosaic show which color corresponds to residuals bigger than the cut-off points $|2|$ and $|4|$ (corresponding to $\alpha=0.05$ and $\alpha=0.0001$, respectively).
The first mosaic (top left, Fig. 6) reveals a significant interaction between Type of transformation and Angle of incidence. Specifically, correct responses occurred more frequently than expected under the independence hypothesis when the movement was orthogonal to the mirror (see the dark gray tile corresponding to the crossing between "correct" and $90^{\circ}$, which has a residual $>2$ ). In contrast, correct responses were significantly less frequent when the movement was at a $67^{\circ}$ angle (see the white tile, corresponding to residuals $<-2$ ); with this angle of incidence, Error type 00 occurred significantly more often (see 
the dark gray tile with thin borders, corresponding to residuals $>4$ ).

A significant interaction was found also between localization and angle of incidence (top right mosaic, Fig. 6). The correct localization was more frequent at $90^{\circ}$ (dark gray tile with thick borders). In contrast, at $67^{\circ}$ angles, correct responses turned out to be less frequent than expected (white tile). With this angle of incidence, participants frequently made endAlign and coPath errors (both tiles are light gray). CoPath errors were also significantly more frequent than expected for the predictions of movements at $45^{\circ}$ (light gray tile).

A third significant two-way interaction was found between Type of transformation and Localization (Fig. 6, bottom mosaic). Correct transformations were usually localized in the correct position (see gray tile with thick borders at the crossing between correct localization and correct responses). With correct responses, the frequency of both types of incorrect localizations was less than expected (see corresponding while tiles with thick borders). Conversely, all three types of errors (OO, II and OI) were frequently associated with localization errors: CoPath errors were significantly associated with all three types of errors (see the dark gray tiles at the bottom of the mosaic), while EndAlign errors frequently occurred in association with Error types $\mathrm{OO}$ and OI (dark gay tiles with thick and normal borders, respectively).

Therefore, although the task was straightforward and the movements were simple, in around $35 \%$ of cases people were unable to make the correct prediction. Errors of types II and OO, which account for the majority of the 35\% erroneous responses, suggest that people follow two heuristics: (1) the reflection and the real object move in the same direction, i.e., they apply the simple rule which holds when a movement is parallel to the mirror or (2) the reflection moves in the opposite direction, i.e., they apply the simple rule which holds when a movement is orthogonal to the mirror.

When the movement was orthogonal, the number of correct responses was particularly high (85\%). The high frequency of Error type 00 with movements at $67^{\circ}$ suggests that the heuristic which assumes that the reflection does the opposite is increasingly evoked as the inclination of the movement gets closer to $90^{\circ}$ (orthogonal). Error type $\mathrm{OO}$ was also associated with the erroneous expectation that the reflection would move along the same line as the real movement (coPath error) or aligned to one endpoint (endAlign error). These factors suggest that participants, when making this error, were thinking about what happens when they approach a mirror at $90^{\circ}$, i.e., that the reflection will move towards them from the opposite direction.

\subsubsection{Confidence ratings}

A paired sample $t$ test between confidence ratings for correct and incorrect transformations did not reveal a difference $(t=$ $1.867 ; d f=50, n s)$.

Experiments 2 and 3 were designed to test the generality of the two heuristics found in Experiment 1 and to exclude the alternative explanation that people think of the reflected world in terms of a $180^{\circ}$ rotation. It is worth noting that the association between Error type $\mathrm{OO}$ and the two errors of localization is compatible with this hypothesis, but that it is not possible to explain Error type II in terms of rotation.

\section{Experiment 2: predicting the orientation of the reflection of a static object in front of a mirror}

To understand whether the beliefs that reflections do the same or do the opposite are specifically related to imagining the dynamics of motion in reflections or, in general, to the imagined spatial correspondence between reflected and real worlds, we tested participants in a condition which was similar to that used in Experiment 1 , but which did not involve motion. Participants were invited to draw the reflection of a fork shape placed in various positions in front of a mirror. We specifically chose a shape with an intrinsic orientation and of a size similar to that of the arrows used in Experiment 1 to allow easy comparison with the results of the previous study.

Bianchi et al. (2008) demonstrated that the perception of opposition between gestures in a reflection and real gestures was more frequent when people observed dynamic rather than static gestures (e.g., moving an arm backward and forward). If opposition is perceived more frequently when movements are involved, it is possible that the idea of opposition comes to mind less often with static reflections. This would mean that there may be fewer errors of the type $\mathrm{OO}$ in this study. However, Bianchi et al. also found that the body orientation (sagittal, gravitational, or coronal) in static reflections was frequently perceived and described as being opposite. A certain number of errors indicating the recall of contrariety patterns might therefore persist.

\subsection{Method}

\subsubsection{Participants}

Twenty-five undergraduates from the Faculty of Educational Sciences, University of Verona (16 females).

\subsubsection{Procedure}

Each participant was presented with nine sheets of paper, in a random order, each containing a schematic drawing of a mirror and a fork shape. They were asked: (a) to draw the reflection of the fork on the other side of the mirror; (b) to rate how confident they were in their response, using an 11-point scale ( 0 to 10) presented at the bottom of each sheet.

\subsubsection{Materials}

Each sheet of paper contained an aerial view of a schematic drawing of a mirror and a fork shape (see Fig. 7). The stimuli varied for the Angle of incidence formed between the main axis of the fork and the mirror $\left(0^{\circ}, 22.5^{\circ}, 45^{\circ}, 67.5^{\circ}, 90^{\circ}\right)$ and the Direction of orientation of the fork (prongs towards the mirror or convergent/ prongs away from the mirror or divergent). For the parallel condition (angle of incidence $=0^{\circ}$ ), we arbitrarily chose to point the prongs of the fork to the right.

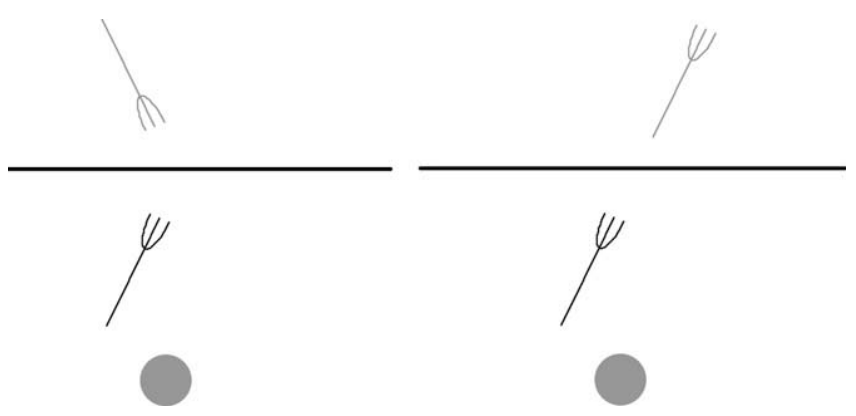

Fig. 7. Two different responses in the paper-and-pencil task in Experiment 2 (participants' responses are represented in gray). The task asked participants to draw the reflected object on the other side of the "mirror" (the black line) as they would see it if they were standing where the gray circle is. A correct transformation in the correct localization is represented in the diagram on the left; Error type II, associated with coPaths error of localization, is represented in the diagram on the right. 


\subsection{Results}

As in Experiment 1, we used generalized (linear) mixed-effect models to test the association between the variables: direction, angle of incidence, type of transformation and localization. No 3-way interactions emerged. We then analyzed all the 2-way interactions using mosaic plots. Because the variable direction (away or towards the mirror) was not significant and was not present in any significant interaction, we will not discuss it in the results.

\subsubsection{Total frequency of correct versus incorrect responses, in terms of} type of transformation or localization

Participants made correct predictions in $72.5 \%$ of cases. The errors (27.5\% of the total number of responses) were Error type II in $19 \%$ of cases and Error type 00 in $7 \%$ of cases; only 2 responses (less than 1\%) were of Error type OI. The localization of the reflection was correctly predicted in $82 \%$ of responses. The incorrect localizations consisted of endAlign errors in $12.4 \%$ of cases and CoPath errors in almost $6 \%$.

\subsubsection{Interactions between variables}

Only two types of two-way interaction turned out to be significant (those shown in the two mosaic plots in Fig. 8). The first interaction was between localization and angle of incidence (left mosaic). At $67^{\circ}$, participants made the coPath error more frequently than would be expected according to the hypothesis that the two variables are independent (see the dark gray tile).

The second interaction was between type of transformation and localization (Fig. 8, right mosaic). All types of errors were significantly associated with erroneous localization. In particular, Error type $\mathrm{OO}$ was associated with both endAligh and coPath errors (see the two corresponding gray tiles with normal borders); the same was found for Error type II (in this case the association with endAlign error has a residual $>4$, see the dark gray tile with thick borders, while that with coPath errors has a residual $>2$, see gray tile with normal border). The few responses showing an Error type OI were associated with endAlign errors. In contrast, for correct transformations, incorrect localizations were significantly fewer than expected (see the two white tiles).

\subsubsection{Confidence ratings}

A paired sample $t$ test between confidence ratings for correct and incorrect transformations did not reveal a difference $(t=1.782 ; d f=13, n s)$.

\subsection{Joint analysis and discussion of Experiments 1 and 2}

Experiment 2 aimed to replicate the results of Experiment 1 using a static instead of a dynamic context, i.e., when the vectors of Fig. 2 are object orientations rather than movements.

The frequencies of the four types of transformation (Correct, Error type OO, Error type II and Error type OI) in the two experiments turned out to be different (Chi-square $=1.860$; Likelihood Ratio $=21.243 ; d f=3, p<.001$ ). As shown by the adjusted residuals in Table 1, the correct responses were more frequent in the static (Experiment 2) than in the dynamic condition (Experiment 1), and Error type $\mathrm{OO}$ was more frequent in the dynamic than in the static condition.

To sum up the results thus far, there are two main outcomes. First, adults made more mistakes when they imagined the movement of a reflection as compared to the orientation of a static ob-
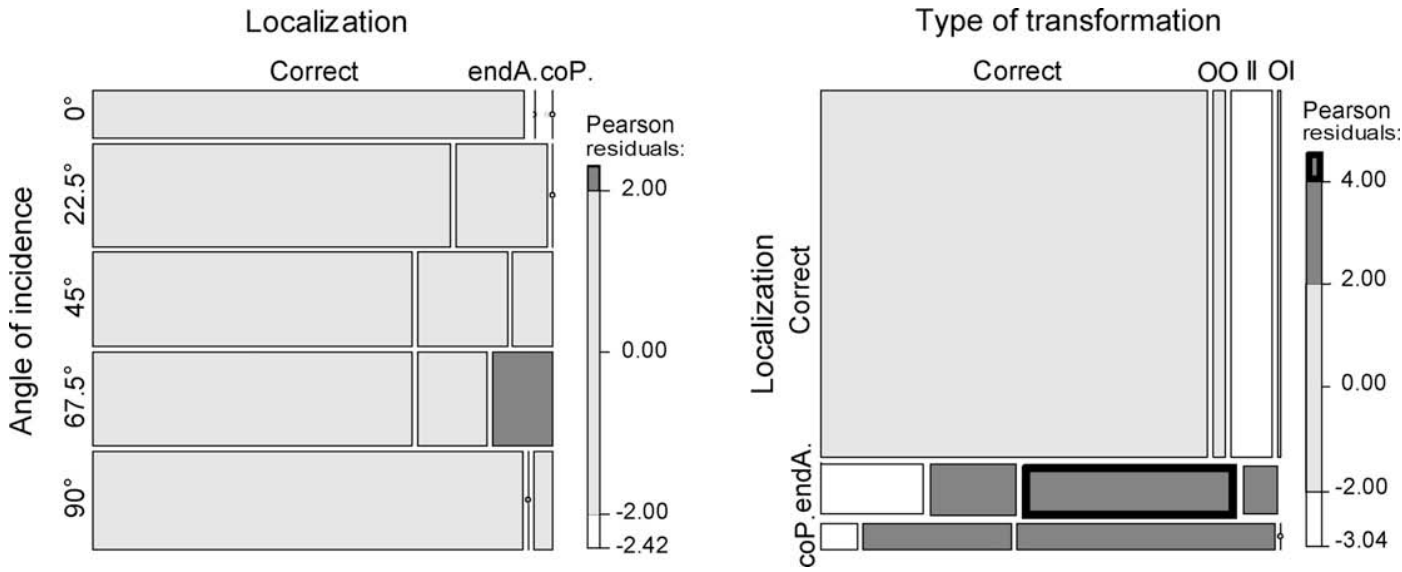

Fig. 8. Mosaic plots representing the two-way interactions which turned out to be significant (under the independence hypothesis, $p<0.001$ ) in Experiment 2. As shown by the bar to the right of each mosaic, dark gray and white tiles (with normal borders) correspond to Pearson residuals greater than $|2|$ (corresponding to $\alpha=0.05$ ); dark gray and white tiles (with thick borders) correspond to residuals greater than $|4|(\alpha=0.0001)$.

Table 1

Frequencies of the four types of transformations in Experiments 1 and 2.

\begin{tabular}{|c|c|c|c|c|c|c|}
\hline & & \multirow[t]{2}{*}{ Correct } & \multicolumn{3}{|c|}{ Type of transformation } & \multirow[t]{2}{*}{ Total } \\
\hline & & & Error OO & Error II & Error OI & \\
\hline \multirow[t]{3}{*}{ Experiment 1: predict the movement of a reflection } & Observed count & 476 & 126 & 107 & 23 & 732 \\
\hline & Expected count & 488.8 & 109.0 & 115.1 & 19.2 & 732.0 \\
\hline & Adjusted residual & -2.1 & 3.7 & -1.7 & 1.8 & \\
\hline \multirow[t]{3}{*}{ Experiment 2: predict the orientation of a reflected static object } & Count & 161 & 16 & 43 & 2 & 222 \\
\hline & Expected count & 148.2 & 33.0 & 34.9 & 5.8 & 222.0 \\
\hline & Adjusted residual & 2.1 & -3.7 & 1.7 & -1.8 & \\
\hline Total & Count & 637 & 142 & 150 & 25 & 954 \\
\hline
\end{tabular}


ject. When they had to imagine a dynamic event they often applied the wrong heuristic.

Second, the types of transformations produced revealed that errors were the result of an oversimplification of the situation in which only one of the two components (identity or opposition) is considered. Moreover, there was little evidence of a confusion between the components because of the low level of error type OI.

In the dynamic condition, the idea that reflections do the same was as frequently applied as the idea that reflections do the opposite, but the latter was, however, more frequently activated as the angle of incidence moved closer to $90^{\circ}$. It was also usually accompanied by localization errors and this supports the hypothesis that Error type $\mathrm{OO}$ is linked to a generalization of how reflections behave with orthogonal movements - which participants, in Experiment 1 , found easier to predict.

The presence of Error type II in both experiments corroborates the idea that people have in mind two heuristics rather than a rotational model. To test this, in Experiment 3 we asked participants to indicate whether they were thinking of reflected movements in terms of identical motion, opposite motion, or rotation. We focused again on dynamic conditions since the two heuristics emerge more clearly in these cases. However, we used an experimental setting that might facilitate the mental re-enacting of how reflections behave.

\section{Experiment 3: predicting motion in reflections while role playing with an imaginary mirror}

In this experiment, we tested whether the type and frequency of errors found in Experiment 1 changed when predictions were made in a more life-like setting as compared to a paper-and-pencil task. Participants were invited to move towards an imaginary mirror, along a straight line at various angles of incidence, and to give directions to another person to make him/her move as they expected a reflection would move.

Participants approached the mirror keeping one arm stretched out in front. This indication of the static spatial orientation of the human body was added to see if participants could cope with dual spatial information and make correct predictions with respect to both these aspects. After a prediction was made, we asked participants to say if they would describe the reflected movement in terms of identical motion, opposite motion or rotation. The aim was to understand whether one of these three transformations better described what they had imagined.

\subsection{Method}

\subsubsection{Participants}

Thirty undergraduate students from the University of Macerata (20 females).

\subsubsection{Procedure}

A strip of white adhesive tape ( $2 \mathrm{~m}$ long) was set on the floor of an empty room. Participants were told to imagine a mirror positioned vertically along this strip. Four paths were marked on the floor, using colored tape corresponding to various angles of incidence: $0^{\circ}$ (parallel to the mirror), $90^{\circ}$ (orthogonal to the mirror), $22^{\circ}$ and $67^{\circ}$. Four starting positions were marked at the beginning of each of these paths. The experimenter invited each participant to stand at one of the starting points and to stretch her/his right arm forwards (this was then repeated for the other three positions; the order of the four starting positions was randomized between subjects). Participants were asked to walk along the colored path on the floor and to indicate how their corresponding reflection would move with the aid of a research collaborator who played the role of the virtual image. Participants directed this person to the correct initial position and then told him/her to move in what they believed would be the correct direction with the correct arm stretched forward.

At the beginning of the experiment the collaborator sat on a chair at a distance of about $3 \mathrm{~m}$ from the imaginary mirror, close to the experimenter, to avoid influencing decisions regarding where the reflection would appear.

After participants had explained how they expected the reflection to move, they were asked to describe the movement of the reflection by indicating whether it was identical or opposite with respect to themselves or rotated through $180^{\circ}$. It was made clear that this question would be repeated for each of the four angles of incidence. It was emphasized that they did not need to worry about being consistent and should choose what they considered the most suitable description in each case.

\subsection{Results}

Generalized (linear) mixed-effect models revealed the absence of 3-way or 4-way interactions between the variables studied (angle of incidence, type of transformation, localization, arm and description). As in Experiments 1 and 2, we analyzed the 2-way interactions using mosaic plots (Fig. 9).

\subsubsection{Marginal frequencies of the four response variables}

Correct versus incorrect responses in terms of type of transformation, localization, which arm was stretched out, and descriptions of the "reflected" movement. In terms of type of transformation, the responses all fall into two categories: they were either correct ( $n=88,73 \%$ ) or involved Error type OO ( $n=29,24 \%)$.

Localization was correct in $73 \%$ of cases $(n=87)$, whereas coPath and endAlign errors were found, respectively, in $18 \%(n=$ $22)$ and less than $2 \%(n=2)$ of responses.

In a similar percentage of cases $(n=88,73 \%)$ participants predicted correctly which Arm was stretched out: they chose the wrong arm in the remaining $26.7 \%$ of cases $(n=32)$.

With respect to the Description of the reflected movement, opposite was considered the best description in $54 \%$ of cases $(n=65)$. The reflection was described as making an identical movement in $35 \%$ of cases $(n=42)$, while in only $8.3 \%$ of cases $(n=10)$ was the movement described in terms of rotation through $180^{\circ}$.

\subsubsection{Interaction between variables}

The four significant two-way interactions are represented in the mosaic plots in Fig. 9.

(i) Type of transformation and localization (Fig. 9, top left). Correct responses in terms of type of transformation were associated with correct localizations (see the large dark gray tile), whereas Error type 00 was associated with coPath errors (dark gray tile with thick borders) and, less frequently, but significantly, with endAlign errors (thin dark gray tile).

(ii) Type of transformation and which arm was stretched out (Fig. 9, top right). Responses predicting that the reflection would stretch out the wrong arm were more frequently found in association with Error type 00 (dark gray tile at the crossing between wrong arm and type of transformation OO).

(iii) Type of transformation and angle of incidence (Fig. 9, bottom left). As in Experiment 1, Error type $\mathrm{OO}$ was particularly frequent when the movement towards the mirror was at a $67.5^{\circ}$ angle (dark gray tile at the crossing between $67^{\circ}$ and type of transformation $\mathrm{OO}$ ). 

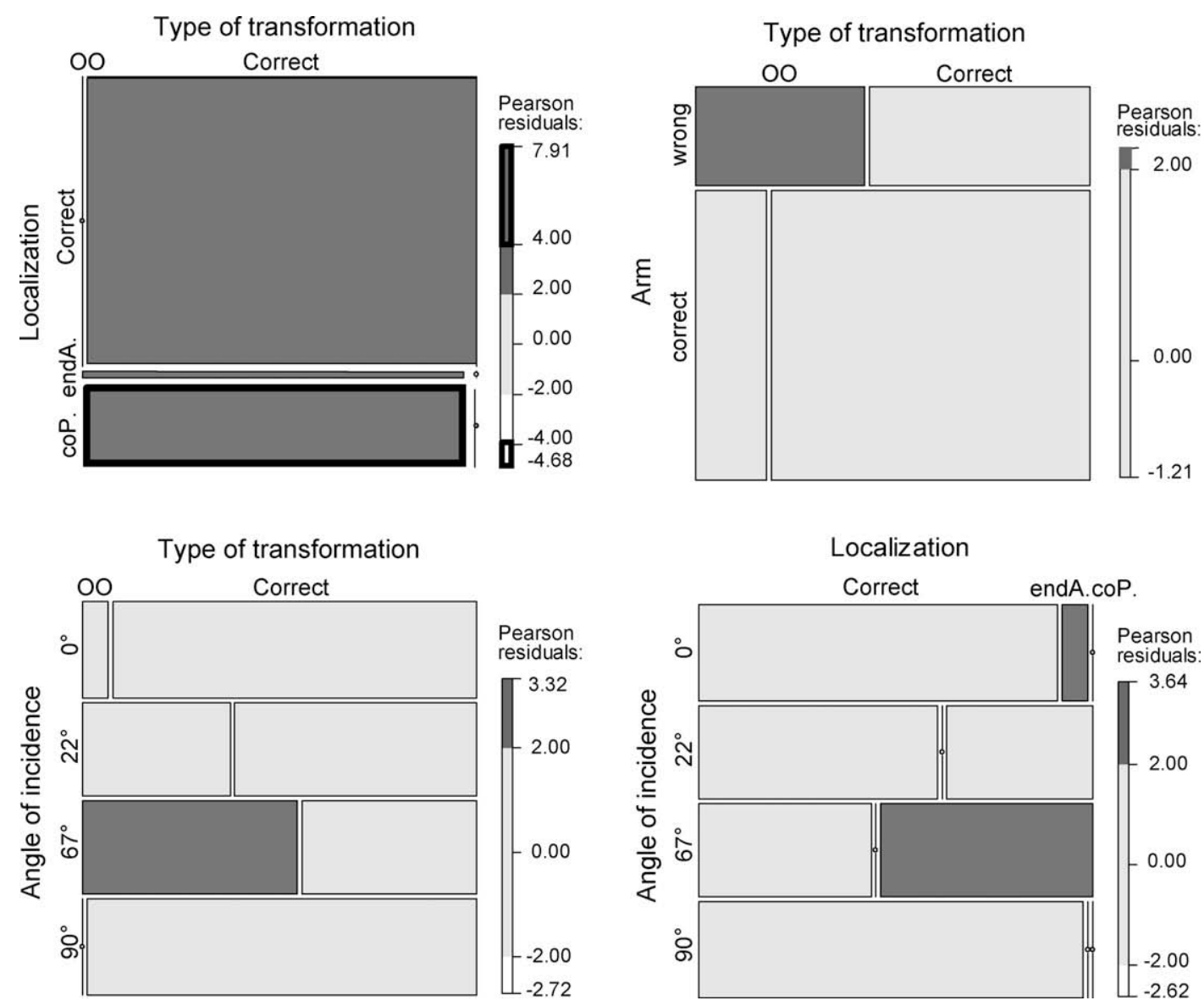

Fig. 9. Mosaic plots representing the two-way interactions which turned out to be significant (under the independence hypothesis, $p<0.01$ ) in Experiment 3 . As shown by the bar to the right of each mosaic, dark gray and white tiles (with normal borders) correspond to Pearson residuals greater than $|2|$ (corresponding to $\alpha=0.05$ ); dark gray and white tiles (with thick borders) correspond to residuals greater than $|4|(\alpha=0.0001)$.

(iv) Localization and angle of incidence (Fig. 9, bottom right). This interaction only involves erroneous localizations: endAlign errors were more frequent with movements parallel to the mirror (dark gray tile at the crossing between $0^{\circ}$ angle and endAlign), while CoPath errors occurred more often with movements at a $67.5^{\circ}$ angle of incidence (gray tile at the crossing between $67^{\circ}$ angle and coPaths).

Experiment 3, in which participants were asked to imagine themselves in front of a mirror, led to fewer errors than the paper-and-pencil task (Experiment 1). However, 25\% of responses were wrong in terms of the type of transformation predicted. Of the two errors which emerged in Experiment 1 (Error types 00 and II), only the former persisted in this more life-like setting, that is, errors resulting from the expectation that reflections do the opposite. Consistently with the previous experiment, this error turned out to be particularly frequent at $67^{\circ}$ angles - in fact this type of error was even more frequent than correct responses and was associated with a coPath error of localization. The fact that we also found an association between Errors of the type $\mathrm{OO}$ and the expectation that the reflection would stretch out the wrong arm (i.e. the opposite arm) further suggest that participants were applying the heuristic that the reflection will do the opposite.

The descriptions given by participants concerning whether the imagined movement is identical, opposite or rotated, confirms the plausibility of the two heuristics regarding identity and opposition - rotation was rarely chosen. The absence of an association between correct or wrong responses and the type of description demonstrates that these two descriptions are generalized and may refer to both correct and incorrect predictions.

\section{Experiment 4: describing the observed behavior of reflections}

So far, we have been interpreting errors as indications that people follow two distinct beliefs or heuristics, i.e., that reflections do the opposite or that reflections do the same. We have also suggested that they are generalizations of what people perceive when they see a reflection of themselves or another person or object.

Experiment 4 tested whether these two patterns (identity and opposition) are grounded in what people perceive when looking at movements or static objects in front of a mirror. Instead of studying human bodies, as in Bianchi et al. (2008), we considered linear movements made by a white ball along the angles of incidence studied in Experiments 1-3, in addition to the static orientation of two objects that differed in terms of their axes of symmetry. The experiment aimed to verify if the relationship perceived between the real movements (or objects) and their reflections complies with the relationship between the vectors described in Fig. 2, namely:

(i) if with movements parallel to the mirror, people perceive the reflection as moving in the same direction as the real movement;

(ii) if with movements orthogonal to the mirror, people perceive the reflection as moving in the opposite direction to the real movement; 
(iii) if movements along the $22^{\circ}$ and $67^{\circ}$ angles of incidence would be described as being either identical or opposite based on their proximity to one of the two "clear-cut conditions" (i.e., parallel motion, when the movement was at $22^{\circ}$, and orthogonal motion, when it was at $67^{\circ}$ ); and

(iv) if the perception of the relationship between the orientation of the reflection of a static object with respect to the real object follow similar rules. If we place a pair of scissors parallel to the mirror so that its elongation is along the horizontal axis (see Fig. 10), we expect people to see the reflection as identical to the real object (because of the absence of asymmetries along the other axes, the $y$ and $z$ vectors do not show any opposition, see Fig. 2). But if we place a puppet (which is front-back, top-bottom and left-right asymmetrical) in front of the mirror, facing the mirror, we are simulating the typical condition of someone facing a mirror.

This condition, potentially, might contribute (a) to the perception of the reflection as opposite the real puppet, because of the opposite exocentric sagittal orientation (opposition along $z$ ); (b) to the perception of the reflection as identical to the puppet, if people assume the mirror surface as the frame of reference of the sagittal orientation (the puppet and its reflection are face to face); (c) to the perception of the reflection as being rotated through $180^{\circ}$, if people compare this configuration with the canonical encounter schema between individuals (in this case, however, participants would have to ignore the fact that the reflected puppet is left-right reversed). In agreement with the results found by Bianchi et al. (2008) and with the hypotheses put forward in this paper, we expected the exocentric orientation to be the most important and that participants would therefore prefer to describe the orientation of the reflected puppet as opposite.

\subsection{Method}

\subsubsection{Participants}

Twenty-five undergraduate students from the University of Macerata (17 females).

\subsubsection{Procedure}

Participants were invited to sit in front of the apparatus shown in Fig. 10. The experimenter presented 6 stimuli (a white ball moving, respectively, at $0^{\circ}, 22^{\circ}, 67^{\circ}$, and $90^{\circ}$; a pair of scissors and a puppet) in a random order. A stick was inserted into the white ball and 4 cuts corresponding to the angles of incidence were made along the top of a box placed in front of the mirror. The experimenter moved the ball towards and away from the mirror by moving the stick under the box along the fissures made by the cuts. The scissors and the puppet were fixed into place as shown in Fig. 10.

Participants observed the objects and their reflections (static or moving, depending on which stimulus was under observation) and then rated their agreement/disagreement with 3 sentences describing the relationship between the reflection and the real object (or movement) as identical, opposite, or rotated through $180^{\circ}$ with respect to the real object (or movement). A seven-point Likert scale (ranging from "totally in agreement" to "totally in disagreement") was printed under each sentence. Participants were shown one stimulus at a time, and the order or the three descriptions to be rated was randomized between participants and between stimuli.

\subsection{Results}

Fig. 11 shows the degree of agreement or disagreement with statements indicating that the reflections were identical, opposite or rotated through $180^{\circ}$ with respect to the real object/movement.

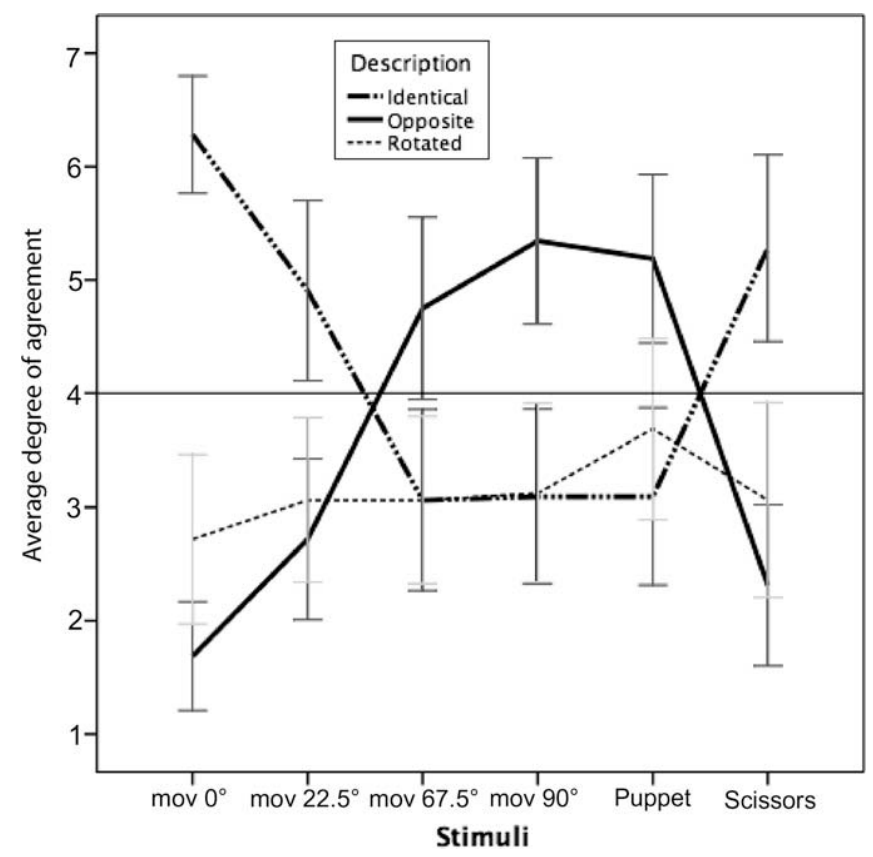

Fig. 11. Average degree of agreement expressed by participants in relation to the three phrases describing the reflection as, respectively, identical, opposite, or rotated with respect to the real object/movement. The ratings (ranging from 1 , totally in disagreement, to 7 , totally in agreement) were given looking at 6 stimuli: two static objects (scissors and puppet) and 4 movements (at, respectively, 0, 22.5, 67.5 and $90^{\circ}$ angles of incidence towards the mirror). Error bars: $\pm 2 \mathrm{SE}$.

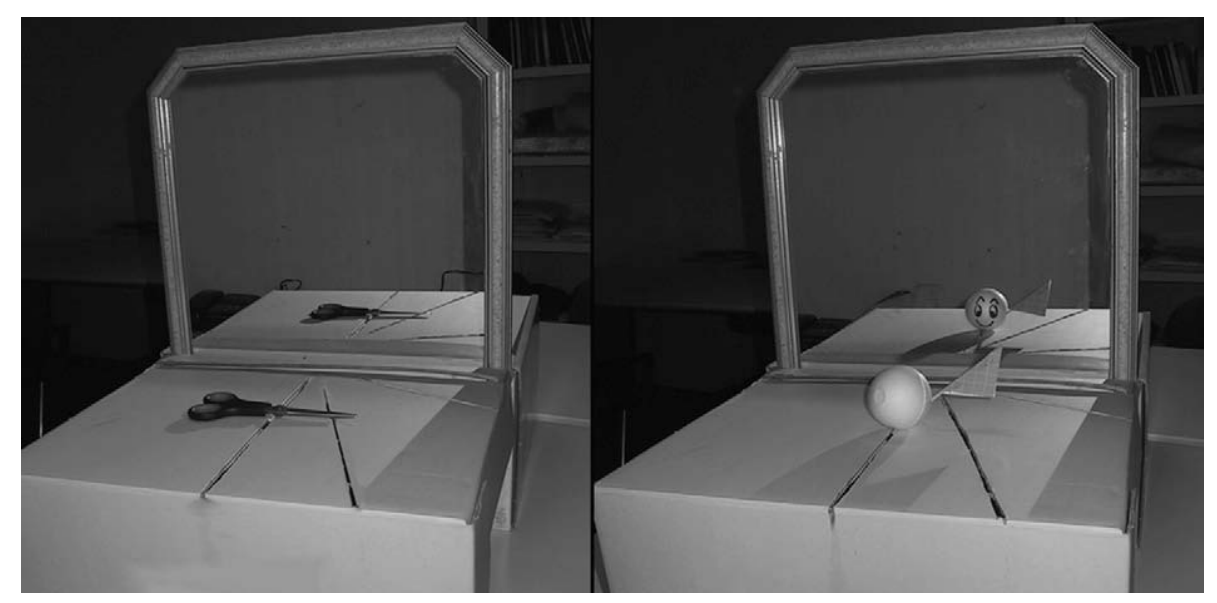

Fig. 10. The apparatus used, with the stimulus "scissors" (picture on the left) and the puppet (picture on the right). 
Descriptions rated at more than 4 points (corresponding to neither in agreement nor in disagreement) indicated:

(i) when the white ball moved parallel to the mirror, or at an angle of incidence of $22^{\circ}$, the movement of the reflection was considered by participants to be best described in terms of identical movement. The same held for the scissors, which were described in terms of identical orientation. For these three conditions the description in terms of opposition was disagreed with;

(ii) when the white ball moved at angles of $67^{\circ}$ and $90^{\circ}$ and when the puppet was in front of the mirror, participants saw the reflection moving (or, in the case of the puppet, oriented) opposite the real object/movement; and

(iii) in none of the cases did participants agreed with a description of the reflection in terms of rotation through $180^{\circ}$.

The findings from Experiment 4 demonstrate an association between what we see and the idea that a reflection does the same or does the opposite. This is consistent with the findings from Experiments 1-3. Moreover, it confirms that identity is perceived in the presence of configurations (real object - reflected object) where the spatial correspondence between the $x$ axes (the parallel component) is salient from a perceptual point of view, whereas opposition is perceived in the presence of configurations where the spatial correspondence between the $z$ axes (the orthogonal component) is salient perceptually. The fact that one object (or movement) is real while the other is virtual is not crucial for this correspondence. Therefore, we expect that similar results would be obtained if the same configurations were composed of two real objects.

\section{Experiment 5: the relationship perceived in "simulated" object-reflection configurations}

To test whether reflected objects are treated perceptually as real objects, as our interpretation of the previous experiments suggests, we conducted a simple variation of Experiment 4. Instead of a real object or movement and its reflection, we used two real objects or movements positioned as if they were an object and its virtual image. The orientation and dynamic behavior of the two objects was identical to those presented in Experiment 4, only this time without a mirror.

\subsection{Method}

\subsubsection{Participants}

Twenty-five undergraduate students from the University of Macerata (16 females).

\subsubsection{Procedure}

Participants sat in front of an apparatus similar to that shown in Fig. 12, except that the mirror had been removed. The experimenter presented 6 pairs of stimuli, each pair with two identical items: two pairs of scissors, two puppets, and two white balls moving at $0^{\circ}, 22^{\circ}$, $67^{\circ}$, and $90^{\circ}$. The experimenter positioned the two static pairs of objects (the scissors or the puppets) and moved the two balls along the cuts made on the top of the box (as in Experiment 4) as if they were an object or movement and its corresponding reflection.

Participants were asked to observe the two objects or movements and then to rate their agreement/disagreement with 3 sentences describing the relationship between them: (a) object B shows an identical orientation/movement with respect to object A; (b) object B shows an opposite orientation/movement with respect to object A; (c) object B is rotated through $180^{\circ}$ with respect to object $A$. The order of presentation of the 6 stimuli was

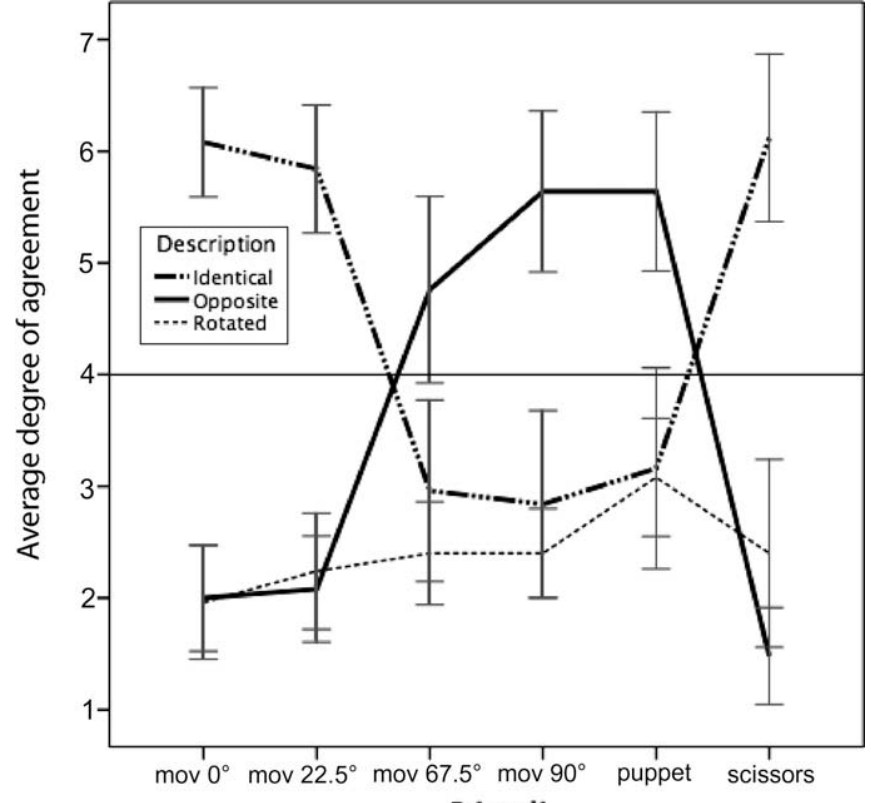

Stimuli

Fig. 12. Average degree of agreement expressed by participants in relation to the three phrases describing the orientation of Object B (scissor, puppet, or white ball moving at $0,22.5,67.5$ and 90 angles of incidence) as, respectively, identical, opposite, or rotated with respect to Object $A$. The ratings ranged from 1 , totally in disagreement, to 7, totally in agreement. Object B was oriented (in the case of the two static objects: scissors and puppet) or moving (in the case of the 4 movements) as if it was the reflection of Object A. Error bars: \pm 2 SE.

randomized between participants, while the order of the 3 descriptions was randomized between stimuli. A seven-point Likert scale (ranging from "totally in agreement" to "totally in disagreement") was printed under each sentence. Participants were asked to observe one stimulus at a time and to express how far they agreed or disagreed with each of the three statements.

\subsection{Results}

The results (see Fig. 12) confirmed what was found in Experiment 4:

(i) When the two balls were moving parallel to each other, or at an angle of incidence of $22^{\circ}$, the movement of ball B was more frequently described as identical with respect to the movement of ball A. The same held for the scissors, where the positioning was described as identical. For these conditions, the description in terms of opposition was considered to be inadequate (mean ratings $<4$ ).

(ii) When the puppets were positioned face to face and the white balls were moved at angles of $67^{\circ}$ and $90^{\circ}$, as if they were on either side of a mirror, participants described the orientation or movement of object $B$ as opposite that of object/movement A.

(iii) In none of the cases did participants agree with a description of the relationship between the two objects or movements in terms of rotation.

\section{Final discussion}

\subsection{Brief overview of the results}

Our experiments aimed to account for predictions made by observers regarding the spatial behavior of reflections, and to 
establish whether these predictions are grounded in what people perceive when looking in mirrors. The focus was on movements, because in this case the geometry of the correspondences between real and virtual worlds is particularly evident. However, we also considered static objects with a similar geometry.

We used a vector description of the spatial relationship between what is on the two sides of a mirror because it complies with observers' experiences and highlights the importance of the role of the exocentric frame of reference in the relationships perceived between real objects and their reflections (Bianchi et al., 2008). Vectorial geometry also clearly shows that identity and opposition are basic features which define the spatial structure of all configurations of real and reflected objects. We expected that if people, when thinking about what happens in mirrors, recall an overall impression of identity and opposition - without analyzing it in terms of the three spatial axes $(x, y$, and $z)$ - then they will generalize patterns of motion which are particularly straightforward in terms of vectorial geometry beyond their conditions of validity (i.e., motion parallel to the mirror and motion orthogonal to the mirror).

The results from the five experiments support this hypothesis. In addition to demonstrating that adults make incorrect predictions (ranging from 25\% of participants in Experiment 3 to 35\% in Experiment 1), our results reveal that the errors conform to two oversimplifications. Participants either expect that the reflection will move in the opposite direction, or in exactly the same direction.

Opposite behavior was more frequently predicted when the task concerned movement (Experiment 1,16.9\%) rather than static orientation (Experiment 2, 7.1\%) and persisted in the case of more life-like conditions (Experiment 3). The percentage of errors was in total lower for life-like conditions (25\%) than in the corresponding paper-and-pencil task (Experiment 1: 35\%), but in the former case all participants that made an error in terms of type of transformation (i.e., 25\%) generalized the expectation that reflections do the opposite.

Participants' descriptions of the imagined movements in Experiment 3 confirmed that they were in effect thinking of opposite motion, not only in the case of Error type 00, but also when describing movements that had been correctly predicted. In other cases, independent of the angle of incidence, participants described the movement as identical.

The last two experiments confirmed the hypothesis that the expectations that reflections will do the same or the opposite are anchored to what people see when looking at reflections (Experiment 4) and, more in general, at configurations where real objects are oriented in the same way as an object and its reflection would be (Experiment 5 ). When observing objects moving parallel or almost parallel to the mirror (namely, at $0^{\circ}$ and $22.5^{\circ}$ ), participants perceived the reflected movement as identical to the real one - and in the condition where both the objects were real, they perceived both objects as carrying out identical movements. An impression of opposition was instead associated with seeing something moving orthogonally $\left(90^{\circ}\right)$ or almost orthogonally $\left(67.5^{\circ}\right)$ towards the mirror (in the case of both genuine and simulated reflections). With static objects (the scissors and the puppet), the description of the reflection as identical or opposite depended on which axis was showing the most evident asymmetry: when it involved the $x$ axis, i.e., parallel to the mirror surface (the scissors), participants described the orientation of the reflection as identical to the real object. When it was along the $z$ axis, i.e., orthogonal to the mirror surface (the puppet), participants described the orientation of the reflection as opposite with respect to the real object. These results were further confirmed when simulated reflections with real objects were considered.
In short, the findings presented in this paper show that participants' responses in predictive tasks are in line with what they see when looking in a mirror. Moreover, they support the claim that identity and opposition are the two spatial relationships which emerge in real-reflected configurations. In this sense, this work also contributes towards the idea that the best geometry for modeling what people have in mind when thinking about the spatial structure of reflections is not based on the geometrical transformations which are normally used to explain mirror symmetry and neither is it an erroneous rotational model. It is instead an identity-opposition structure.

\subsection{From perceptual experience to naïve beliefs}

Our findings support the literature that has investigated the role of perceptual experience in the field of naïve physics or naïve optics. Lawson and Bertamini (2006) explicitly questioned whether errors made by adults concerning mirror phenomena should be considered as conceptual or perceptual, in other words, whether wrong beliefs were founded on what observers see (and in this case it should not be surprising that familiarity does not improve performance) or whether they were purely conceptual. In this last case, errors should disappear when direct perceptual support is provided. Lawson and Bertamini, found that the early error (i.e., the expectation that when moving parallel to a mirror, one would see oneself reflected before one actually does) was eliminated by providing visual feedback. They therefore concluded that this type of error was conceptual. Other errors, in contrast, persisted even when visual feedback was provided; for example, people overestimated the size of reflections on the surface of mirrors, even when they were given visual feedback. This last result was used to support the claim that this type of error was caused by a powerful perceptual phenomenon.

The attempt to discover the perceptual versus conceptual foundation of erroneous naïve beliefs is not specific to naïve optics, but has also been a more general issue in the field of naïve physics. Different authors have proved the perceptual foundation of some beliefs about physical phenomena.

(i) In 1958, Bozzi demonstrated that when presented with a swinging pendulum, people perceived only some of the swings as natural while others were perceived as too fast or too slow. He also showed that they followed this perceptual law rather than the isochronism law when they were asked to adjust the velocity of the oscillation of the pendulum.

(ii) Bozzi (1959) and Runeson (1974) demonstrated that when asked to indicate the correct simulation of a something falling down an inclined plane, people did not choose the one that seemed to be accelerating but one whose movement seemed to be uniform (although this latter physically started with a brief acceleration and then became uniform). In everyday viewing conditions people do not notice any acceleration in falling objects, but see them as falling at a uniform speed. No surprise therefore that they chose a simulation of motion which they perceived to be uniform.

(iii) McCloskey et al. (1983) found that even when participants were shown animations of the natural, parabolic trajectories of objects dropped from a moving carrier, many people indicated that the objects were falling straight down. The moving carrier functioned as a frame of reference for the falling object and this transformed the perception of a parabolic trajectory into a fall that went straight down. Again, no surprise therefore to discover that people (in predictive tasks) manifest the belief that objects fall straight down and not following a parabolic trajectory. 
(iv) Sholl and Liben (1995) reported that when shown water in a tilted container, people misperceived the water surface as tilted. In this case too, a perceptual experience can be the origin of mistaken beliefs concerning the fact that the surface of a liquid in a container would tilt (and not remain parallel to the ground) when the container itself is tilted (McAfee \& Profitt, 1991).

A common characteristic of these various false beliefs, which are consistent with perceptual experience, is that they persist even after the correct physical behavior had been explained as happened in the case of the overestimation error in naïve optics, mentioned by Lawson et al. (2007) as an example of perceptual error.

\subsection{Two levels of perceptual grounding of naïve beliefs}

We think that the idea held by Yates et al. (1988) regarding prototypical events provide an interesting extension of the area of influence of perception on naïve beliefs. It not only accounts for cases where beliefs are consistent with what people perceive in specified situations, but it also accounts for cases where people generalize real perceptions beyond the specific conditions in which they occur. The results presented in this paper support this. We cannot say that the errors made by our participants are founded in perception in the same way as the phenomena mentioned above in points (i)-(iv) can be said to be based on what people see. In reality, nobody has ever seen a reflection moving as represented in the second, third and fourth columns of Fig. 4. These errors demonstrate the fact that people, when thinking of or imagining the behavior of reflections, recall an overall impression of identity and contrariety or precise perceptual experiences where they are particularly evident. They recall what can be thought of in terms of the prototypical behavior of reflections (what is seen when moving parallel or orthogonal to a mirror) and apply them to circumstances in which the resultant vector needs to be taken into account. So the point is not that they do not see a certain aspect - as in the cases mentioned above in points (i)-(iv) - but that only part of what they see becomes salient, functioning as a prototypical structure in the absence of a corresponding perceptual event.

Note that a similar explanation might account for the abovementioned early error (Bertamini et al., 2003), which cannot be explained as a perceptual error. When people walk parallel to a mirror they start seeing their reflection when their eyes reach the closest edge of it. In this sense there is no perceptual foundation to the expectation of seeing oneself before. This error can, however, be founded in perception according to the prototype view. When looking in mirrors intentionally or occasionally in everyday life, the eyes of the observer are not usually aligned with the near edge of the mirror and the attention is rarely focused on what happens in this area. It is certainly more common for observers to find themselves in different positions with respect to the mirror (directly in front of or beyond its edges) and so their line of vision is incident to the mirror at different angles. In each case, large portions of the real world which is beyond the edges of the mirror are visible. This is what people normally perceive. If they are not careful to distinguish between the behavior of reflections at the near and far edges of a mirror, this experience of a mirror reflecting the physical world beyond its boundaries likely becomes the prototypical view and participants would think of it in prediction tasks. The generalization to incorrect instances of correct perceptions might therefore also explain the early error in naïve optics. We are inclined to believe that this mechanism could in addition explain many other errors in naïve physics.

\section{Acknowledgments}

We would like to thank Michael Kubovy and Heiko Hecht for their comments on an earlier version of this paper.

\section{References}

Bertamini, M., Lawson, R., \& Liu, D. (2008). Understanding 2D projections on mirrors and on windows. Spatial Vision, 21, 273-289.

Bertamini, M., \& Parks, T. E. (2005). On what people know about images on mirrors. Cognition, 98, 85-104.

Bertamini, M., Spooner, A., \& Hecht, H. (2003). Naïve optics: Predicting and perceiving reflections in mirrors. Journal of Experimental Psychology: Human Perception and Performance, 29(5), 982-1002.

Bianchi, I., \& Savardi, U. (2008). The relationship perceived between the real body and the mirror image. Perception, 5, 666-687.

Bianchi, I., Savardi, U., \& Bertamini, M. (2008). Estimation and representation of head size (people overestimate the size of their head - evidence starting from the 15th century). British Journal of Psychology, 99(4), 513-531.

Bozzi, P. (1958). Analisi fenomenologica del moto pendolare armonico. Rivista di Psicologia, 52(4), 281-302 (P. Bressan, P. Gaudiano, Transl.), Phenomenological Analysis of Pendular Harmonic Motion. Little Rock, AR 72204, USA: Department of Psychology, UALR, 2801 South University, 1989.

Bozzi, P. (1959). Le condizioni del movimento "naturale" lungo i piani inclinati. Rivista di Psicologia, LIII(II) 337-352 (P. Bressan, P. Gaudiano, Transl.), The Conditions for "Natural" Motion Along Inclined Planes. Little Rock, AR 72204, USA: Department of Psychology, UALR, 2801 South University, 1989.

Croucher, C. J., Bertamini, M., \& Hecht, H. (2002). Naïve optics: Understanding the geometry of mirror reflections. Journal of Experimental Psychology: Human Perception and Performance, 28, 546-562.

Frick, A., Huber, S., Reips, U., \& Krist, H. (2005). Task-specific knowledge of the law of pendulum motion in children and adults. Swiss Journal of Psychology, 64(2), 103-114.

Friendly, M. (1994). Mosaic displays for multi-way contingency tables. Journal of the American Statistical Association, 89, 190-200.

Friendly, M. (2000). Visualizing categorical data. Carey, NC: SAS Institute. URL http:// www.math.yorku.ca/SCS/vcd/.

Hecht, H., \& Bertamini, M. (2000). Understanding projectile acceleration. Journal of Experimental Psychology: Human Perception and Performance, 26, 730-746.

Hecht, H., Bertamini, M., \& Gamer, M. (2005). Naïve optics: Acting upon mirror reflections. Journal of Experimental Psychology: Human Perception and Performance, 31, 1023-1038.

Kaiser, M. K., Proffitt, D. R., \& McCloskey, M. (1985). The development of beliefs about falling objects. Perception and Psychophysics, 38, 533-539.

Lawson, R., \& Bertamini, M. (2006). Errors in judging information about reflections on mirrors. Perception, 35, 1265-1288.

Lawson, R., Bertamini, M., \& Liu, D. (2007). Overestimation of the projected size of objects on the surface of mirrors and windows. Journal of Experimental Psychology: Human Perception and Performance, 33, 1027-1044.

McAfee, E. A., \& Profitt, D. R. (1991). Understanding the surface orientation of liquids. Cognitive Psychology, 23, 122-130.

McCloskey, M. (1983). Intuitive physics. Scientific American, 248, 122-130.

McCloskey, M., Caramazza, A., \& Green, B. (1980). Curvilinear motion in the absence of external forces: Naïve beliefs about the motion of objects. Science, 210, 1139-1141.

McCloskey, M., Washburn, A., \& Felch, L. (1983). Intuitive physics: The straightdown belief and its origin. Journal of Experimental Psychology: Learning, Memory, and Cognition, 9, 636-649.

Meyer, D., Zeileis, A., \& Hornik, K. (2006). The Strucplot framework: Visualizing multi-way contingency tables with vcd. Journal of Statistical Software, 17(3), $1-48$

Pittenger, J. B. (1989). Detection of violation of the law of pendulum motion: Observers' sensitivity to the relation between period and length. Ecological Psychology, 2, 55-81.

Proffitt, D. R. (1999). Naïve physics. In R. Wilson \& F. Keil (Eds.), The MIT encyclopedia of the cognitive sciences (pp. 577-579). Cambridge, MA: MIT Press.

Proffitt, D. R., \& Gilden, D. L. (1989). Understanding natural dynamics. Journal of Experimental Psychology: Human Perception and Performance, 15, 384-393.

Proffitt, D. R., \& Kaiser, M. K. (2003). Intuitive physics. In L. Nadel (Ed.), Encyclopedia of cognitive science (pp. 632-637). London: Macmillan.

Profitt, D. R., Kaiser, M. K., \& Whelan, S. M. (1990). Understanding wheel dynamics. Cognitive Psychology, 22, 342-373.

Runeson, S. (1974). Constant velocity - not perceived as such. Psychological Research, 37, 3-23. 
Savardi, U., \& Bianchi, I. (2005). Looking at yourself in the mirror: Structures of perceptual opposition. Gestalt Theory, 3, 204-220.

Shaffer, D. M., \& McBeath, M. K. (2005). Naïve beliefs in baseball: Systematic distortion in perceived time of apex for fly balls. Journal of Experimental Psychology: Learning, Memory and Cognition, 31(6), 1492-1501.

Shanon, B. (1976). Aristotelianism, Newtonianism and the physics of the Layman. Perception, 5, 241-243.

Shepard, R. N., \& Cooper, L. A. (1982). Mental images and their transformations. Cambridge, MA: MIT Press/Bradford Brooks.
Sholl, M. J., \& Liben, L. S. (1995). Illusory tilt and Euclidean schemes as factors in performance on the water-level task. Journal of Experimental Psychology: Learning, Memory and Cognition, 21, 1624-1638.

Yates, J., Bessman, M., Dunne, M., Jertson, D., Sly, K., \& Wendelboe, B. (1988). Are conceptions of motion based on a naïve theory or on prototypes? Cognition, 29 , 251-275. 